\title{
In-Hospital Mortality Risk Model of Gastric Cancer Surgery: Analysis of a Nationwide Institutional-Level Database With 94,277 Chinese Patients
}

\section{OPEN ACCESS}

Edited by:

Gyu Seog Choi,

Kyungpook National University,

South Korea

Reviewed by:

Zahir Soonawalla

Oxford University Hospitals NHS Trust,

United Kingdom

Paul Willemsen,

Ziekenhuisnetwerk Antwerpen Middelheim, Belgium

*Correspondence:

Ziyu Li

ziyu_li@hsc.pku.edu.cn

Jiafu Ji

jiijafu@hsc.pku.edu.cn

tThese authors have contributed equally to this work

Specialty section

This article was submitted to

Surgical Oncology,

a section of the journal

Frontiers in Oncology

Received: 28 May 2019 Accepted: 19 August 2019 Published: 01 October 2019

Citation:

Wu Z, Cheng $H$, Shan $F$, Ying $X$ Miao $R$, Dong J, Sun $Y, X u A$, Zhou $Y$, Wang Y, Chen L, Xue Y, Cao H, Hua Y,

$X u Z$, Zheng $M$, Yan $M$, Huang $C$,

Suo J, Liang H, Fan L, Hu J, Hu X,

LiG, Yu P, Li G, Shi Y, Luo H, Li Y, Xie M, Liu T, Zhang Z, Shi T, Li Z and Ji J (2019) In-Hospital Mortality Risk Model of Gastric Cancer Surgery:

Analysis of a Nationwide

Institutional-Level Database With 94,277 Chinese Patients.

Front. Oncol. 9:846. doi: 10.3389/fonc.2019.00846
Zhouqiao Wu ${ }^{1+}$, Huimin Cheng ${ }^{2 \dagger}$, Fei Shan ${ }^{1}$, Xiangji Ying ${ }^{1}$, Rulin Miao ${ }^{1}$, Jianhong Dong ${ }^{3}$, Yihong Sun ${ }^{4}$, Aman Xu ${ }^{5}$, Yanbing Zhou ${ }^{6}$, Yanong Wang ${ }^{7}$, Lin Chen ${ }^{8}$, Yingwei Xue ${ }^{9}$, Hui Cao ${ }^{10}$, Yawei Hua ${ }^{11}$, Zekuan Xu ${ }^{12}$, Minhua Zheng ${ }^{10}$, Min Yan ${ }^{10}$, Changming Huang ${ }^{13}$, Jian Suo ${ }^{14}$, Han Liang ${ }^{15}$, Lin Fan ${ }^{16}$, Jiankun $\mathrm{Hu}^{17}$, Xiang $\mathrm{Hu}^{18}$, Guoli $\mathrm{Li}^{19}$, Peiwu Yu${ }^{20}$, Guoxin $\mathrm{Li}^{21}$, Yiran Shi ${ }^{22}$, Huayou $\mathrm{LuO}^{23}$, Yong $\mathrm{Li}^{24}$, Ming Xie ${ }^{25}$, Tianxue $\mathrm{Liu}^{26}$, Zhongyuan Zhang ${ }^{2}$, Ting Shi ${ }^{27}$, Ziyu $\mathrm{Li}^{1 *}$ and Jiafu $\mathrm{Ji}^{1 *}$ on behalf of China Gastrointestinal Cancer Surgery Union

${ }^{1}$ Key Laboratory of Carcinogenesis and Translational Research (Ministry of Education), Department of Gastrointestinal Surgery, Peking University Cancer Hospital and Institute, Beijing, China, ${ }^{2}$ School of Statistics and Mathematics, Central University of Finance and Economics, Beijing, China, ${ }^{3}$ Center of Minimally Invasive Gastrointestinal Surgery, Shanxi Provincial Cancer Hospital, Taiyuan, China, ${ }^{4}$ Department of General Surgery, Fudan University Zhongshan Hospital, Shanghai, China, ${ }^{5}$ Department of General Surgery, The First Affiliated Hospital of Anhui Medical University, Hefei, China, ${ }^{6}$ Department of General Surgery, The Affiliated Hospital of Qingdao University, Qingdao, China, ${ }^{7}$ Department of Gastric Cancer and Soft Tissue Sarcomas, Fudan University Shanghai Cancer Hospital, Shanghai, China, ${ }^{8}$ Department of General Surgery, People's Liberation Army General Hospital, People's Liberation Army (PLA) Army General Hospital, Beijing, China, ${ }^{9}$ Department of Gastrointestinal Surgery, Harbin Medical University Cancer Hospital, Harbin, China, ${ }^{10}$ Department of General Surgery, Ruijin Hospital, Shanghai Jiao Tong University, Shanghai, China, ${ }^{11}$ Department of General Surgery, Henan Cancer Hospital, Zhengzhou, China, ${ }^{12}$ Department of General Surgery, The First Affiliated Hospital of Nanjing Medical University, Nanjing, China, ${ }^{13}$ Department of Gastric Surgery, Fujian Medical University Union Hospital, Fuzhou, China, ${ }^{14}$ Department of Gastrointestinal Surgery, The First Hospital of Jilin University, Changchun, China, ${ }^{15}$ Department of Gastric Cancer, Tianjin Medical University Cancer Institute and Hospital, Tianjin, China, ${ }^{16}$ Department of General Surgery, The First Affiliated Hospital of Xi'an Jiaotong Hospital, Xi'an, China, ${ }^{17}$ Department of Gastrointestinal Surgery, West China Hospital, Chengdu, China, ${ }^{18}$ Department of General Surgery, The First Affiliated Hospital of Dalian Medical University, Dalian, China, ${ }^{19}$ Department of Gastrointestinal Surgery, Nanjing General Hospital, Nanjing, China, ${ }^{20}$ Department of General Surgery, The First Hospital Affiliated Hospital to Army Medical University (AMU) (Southwest Hospital), Chongqing, China, ${ }^{21}$ Department of General Surgery, Nanfang Hospital, Guangzhou, China, ${ }^{22}$ Department of General Surgery, Weifang People's Hospital, Weifang, China, ${ }^{23}$ Department of Gastrointestinal Surgery, First Affiliated Hospital of Kunming Medical University, Kunming, China,

${ }^{24}$ Department of General Surgery, Guangdong General Hospital, Guangzhou, China, ${ }^{25}$ Department of General Surgery, Affiliated Hospital of Zunyi Medical University, Zunyi, China, ${ }^{26}$ Department of General Surgery, Penglai People's Hospital, Penglai, China, ${ }^{27}$ Centre for Global Health Research, Usher Institute of Population Health Sciences and Informatics, University of Edinburgh, Edinburgh, United Kingdom

Background: The objective of this study is to identify independent risks and protective factors and to construct a mortality prediction model for gastrectomy in the Chinese population.

Study design: This is a population-based prospective cohort at an institutional level. Seventy-two participating hospitals reported their annual gastrectomy data between 2014 and 2016, while 44 variables covering the institution and surgical information were included in the analysis. We used $\mathrm{R}$ software to encode and complete data pre-processing. The first difference model was applied to build the risk model. Data from 2014 and 2015 were assigned to risk model development, while data from 2016 was used for validation. 
Results: In the included centers with 94,277 gastric cancer cases, the in-hospital mortality rate was $0.32 \%$. The regression model revealed that provinces with low-middle GDP, hospitals with annual gastrectomy volume between 100 and 500, greater volume of urgent surgeries performed, larger proportion of males, and a higher proportion of liver metastasis were independent risk factors for mortality following gastric surgeries, while higher laparoscopic resection volume, greater volume of distal gastrectomy with B2 reconstruction, and larger proportion of palliative surgery were independent protective factors $(p<0.05$, respectively). In the prediction test, the mean square error of the training set was 0.948 , while that of the test set was 0.728 , demonstrating the effectiveness of this model.

Conclusions: We constructed the first mortality risk prediction model for gastric cancer surgery in the Chinese population. The identified risk factors will help with the therapy selection, while further informing Chinese medical policy decision-makers.

Keywords: gastric cancer, surgical safety, mortality, national database, prediction model

\section{INTRODUCTION}

Gastric cancer is still a common global malignancy with over one million new cases each year, where more than half occurs in China. It remains the second most commonly diagnosed cancer in the ethnic Chinese population (1). Although early stage cases can be treated with endoscopic therapy, the majority of cases are already locally advanced at the time of diagnosis, in which case, surgical resection is obligatory for the treatment. Despite the steady improvement of oncological survival in the recent decades, surgical complications after gastrectomy are still fairly common (18.3-36\%) (2-4). Major complications including anastomotic leakage, peritonitis, bleeding, and ileus could significantly hinder postoperative recovery, or even threaten the patient's life if not properly managed (5). Mere delays in recovery also postpone adjuvant therapy, which may influence oncological survival, let alone intra-abdominal infectious complications, which are associated with earlier recurrence and reduced longterm survival rates $(6,7)$.

To reduce postoperative mortality and complications, the medical infrastructure has seen a rise in the number of national surgical databases, producing invaluable findings to inform both domestic and international medical communities (8-10). The China Gastrointestinal Cancer Surgical Union was founded in 2016 for the purpose of improving surgical quality and safety in China by documenting and sharing cases. As the first step, we collected the annual summary of 72 participating medical centers with nearly 200,000 gastric and colorectal patient data in China between 2014 and 2016, and created the China Gastrointestinal Surgery Database (CGSD). Using this database, we constructed the first mortality prediction model for gastrectomy in the Chinese population.

\section{METHODS}

\section{CGSD Database}

The CGSD was established for risk factor stratification of gastric and colorectal cancer surgery in the Chinese population.
All member institutions must submit its annual institutional summary data to the central database. The CGSD's final structure is expected to be similar to the ACS-NSQIP (American College of Surgeons-National Surgical Quality Improvement Program) and the Japanese NCD (National Clinical Database) programs $(2,9)$, which yield a collection of individual-level data in the form of patients' demographic characteristics, pre-existing comorbidities, pre-operative laboratory results, surgical details, and postoperative outcomes. The majority of the participating centers are tertiary hospitals, covering $88.2 \%$ (30/34) provinces of China, with 94,277 gastric and 90,076 colorectal cancer patients' data collected between 2014 and 2016.

One of the ultimate goals of the CGSD is to predict institutional mortality, we therefore developed a prediction model in this study. We assigned data from 2014 and 2015 to the risk model development (training set), while data from 2016 was used for prediction model validation (test set).

\section{Data Collection}

Each participating institution reported their annual summary data of gastric cancer surgeries. In total, 44 variables were included in the analysis, including institution information (e.g., hospital name, hospital type, annual gastrectomy volume, number of surgeons, and beds in the team etc.), and surgical information (e.g., number of open and laparoscopic gastrectomy, number of total, distal and proximal gastrectomy, number of different reconstruction types, number of surgery after neoadjuvant therapy, number of palliative surgery etc.).

\section{Endpoints of the Study}

In this study, we used in-hospital mortality as our primary endpoint. It was defined as death during the hospitalization of the surgical treatment, regardless of the length of hospital stay or cause of death. In addition, the other safety parameters including re-operation rate and post-operative stay were selected as the secondary endpoints. 


\section{Data Analysis}

We used R (Version 3.3.2) software to encode and complete data pre-processing automatically. For the missing data, we imputed them by applying K-nearest neighbor (KNN). The proportion of missing data were $<10 \%$ of all included variables, and most variables $(40 / 44)$ had $<5 \%$ of missing data (Table S1). The 3 year average of variables of interest were described as mean (min. to max). If not indicated, the averages were weighted by center volume.

In the training set, we used the first difference model for the prediction model, because it could circumvent the issue of non-independence of repeated measurements in each center. This model has been frequently used for the purposes of trend prediction in repeated measurements (11). The model can be specified as:

$$
\Delta y=\sum_{i=1}^{k} \beta_{i} \Delta x_{i}+\Delta u
$$

where $\Delta y$ and $\Delta x_{i}$ denote the first difference in response and predictors respectively. In other words, $\Delta y_{t}=y_{t}-y_{t-1}, \Delta x_{i t}=$ $x_{i t}-x_{i t-1}$, where $t$ represents time, that is, different years of this study. $\beta_{i}$ represents the influence of a unit of change in $\Delta x_{i t}$ on $\Delta y_{t}(12,13)$, and $e_{t}$ denotes the residual. For example, $\beta_{i}=3$ means that if $x_{i}$ increases by 1 unit at time t, then $y$ will increase by 3 units at time $t$.

In the section of prediction validation (test set), mean square error (MSE) is applied to evaluate the accuracy of the model prediction, which is calculated as below:

$$
\mathrm{MSE}=\frac{1}{N} \sum_{i=1}^{N}\left(Y_{i}-\hat{Y}_{i}\right)^{2},
$$

where $Y_{i}$ and $\hat{Y}_{i}$ denotes the observed and predicted value $\left(Y_{i}-\right.$ $\left.\hat{Y}_{i}\right)$, is known as residual. Lower MSE means better prediction accuracy. An MSE of zero, meaning that the estimators $\hat{Y}_{i}$ predicts observations $Y_{i}$ with perfect accuracy.

All data analyses were conducted using $\mathrm{R}$ (version 3.3.2). A two-sided $P$ value $<0.05$ is considered as statistical significance. No ethical approval nor informed consent was required for this study under the domestic legislation.

\section{RESULTS}

\section{Demographic Characteristics}

There are 72 hospitals reported the gastric cancer data to the database. Among them, $78 \%$ are general hospitals while the other $22 \%$ are cancer hospitals.

The average annual volume of gastric cancer surgery per institution was 585, which was conducted by an average of 9.6 surgeons per hospital. The average volume of open gastrectomy was 260.1 cases per year, while that of the laparoscopic ones was 135.2 cases per year. The average number of harvested lymph nodes was 27.4. A summary of the demographic characteristics is listed in Table 1.

\section{Mortality}

The in-hospital mortality rate was $0.3 \%(0-3.6 \%)$, and the other surgical safety outcomes are summarized in Table 2.

\section{Predictive Factors}

The regression model revealed that low/middle GDP provinces, annual gastrectomy volume between 100 and 500, greater numbers of urgent surgeries performed due to bleeding or obstruction, higher reoperation rate, greater proportion of male patients, and a greater proportion of liver metastasis were independent risk factors for mortality after gastric surgery, while higher laparoscopic resection volume, higher distal gastrectomy with B2 reconstruction volume, and higher proportion of palliative surgery were the independent protective factors $(p<0.05$ respectively, Table 3$)$. All risks and protective factors listed above were measured at an institutional level.

\section{Prediction Model Evaluation}

The gastric mortality-risk prediction model shows that the MSE of the training set was 0.948 , while that of the test set was 0.728 . Most residuals in the model are concentrated near 0 . The low MSE in the model shows the effectiveness of our predictive model (Figure 1).

\section{DISCUSSION}

This is the first report, based on the large-scale national surgical database that we have recently established, of institution-level risk stratification and in-hospital mortality prediction on gastric cancer surgeries in China.

The in-hospital mortality rate of gastric cancer surgery was $0.32 \%$ in our database, varying between 0 and $3.6 \%$ in different participating centers. This is comparable to the results from other Asian countries (0.3-1.2\%) (3, 14), and seems lower than some studies from Europe or America (4.7-10\%) $(2,8,15)$. However, such difference cannot simply be explained by surgical quality or safety. One obvious reason is that the disease epidemiology (including severity) varied across regions, which substantially varied the surgical difficulties in different areas. Another possible explanation is that many other national databases also include emergency surgery with non-malignant causes, which may substantially increase the mortality [0.7 vs. $6.0 \%$ for right hemicolectomy (16)], while our database primarily focuses on cases of cancer. Although some urgent cases due to bleeding or obstruction were still performed (and those were indeed identified as risk factors in the results), the majority of the cases were elective procedures.

We chose in-hospital mortality rather than the 30-day mortality (a more frequently used parameter for surgical safety evaluation) as the primary endpoint in CGSD. This is because of the currently unsatisfactory follow-up system. In China, most gastric cancer patients are treated in tertiary hospitals in the cities (17), however many of the patients continue their followup treatment in the primary hospitals that are distant from the cities. In this case, it is difficult to obtain follow-up data updates, therefore, in-hospital mortality is a more practical and reliable parameter to evaluate the surgical safety in the current circumstances.

Many of our identified risk factors were also reported in the previous literature, such as urgent surgery (bleeding, obstruction) (4), and greater male proportion (3). In addition, 
TABLE 1 | Summary of demographic characteristics.

\begin{tabular}{|c|c|c|c|c|c|}
\hline Parameter & Mean & Mean' & Min & Max & Preprocessing \\
\hline Provincial GDP level & - & - & - & - & $\begin{array}{l}\text { Divided into } 4 \text { categories in decreasing order by the } \\
\text { GDP of the hospital: } 34.72,33.33,16.67,15.28 \%\end{array}$ \\
\hline Hospital Type & - & - & - & - & $\begin{array}{l}\text { Divided into: General hospital (78\%), Cancer } \\
\text { hospital (22\%) }\end{array}$ \\
\hline Male proportion & $68.88 \%$ & $68.97 \%$ & $46.09 \%$ & $81.41 \%$ & Number of Male patients/Number of all patients \\
\hline Average Age & 59.86 & 59.71 & 52.57 & 67.06 & \\
\hline Number of beds for gastric cancer surgery & 78.76 & 80.77 & 15 & 328 & \\
\hline Number of gastric cancer surgeons & 9.64 & 9.79 & 2 & 40 & \\
\hline Average surgeon per bed & 0.14 & 0.14 & 0.03 & 0.67 & Number of surgeons/number of beds \\
\hline Annual gastrectomy volume level & - & - & - & - & $\begin{array}{l}\text { Divided into } 3 \text { levels: <100(9.09\%), } \\
100-500(44.16 \%),>500(46.75 \%)\end{array}$ \\
\hline $\begin{array}{l}\text { Urgent surgery volume due to bleeding } \\
\text { or obstruction }\end{array}$ & 25.49 & 25.41 & 2 & 53 & \\
\hline Open gastrectomy volume & 260.12 & 267.30 & $0^{*}$ & 1277 & \\
\hline Open total gastrectomy volume & 113.02 & 115.7 & $0^{*}$ & 771 & \\
\hline Open distal gastrectomy volume & 134.73 & 138.76 & $0^{*}$ & 495 & \\
\hline Open proximal gastrectomy volume & 29.12 & 29.02 & $0^{*}$ & 345 & \\
\hline Laparoscopic resection volume & 135.17 & 118.95 & $0^{\star}$ & 614 & \\
\hline Laparoscopic total gastrectomy volume & 52.66 & 46.30 & $0^{*}$ & 464 & \\
\hline Laparoscopic distal gastrectomy volume & 77.44 & 68.45 & $0^{*}$ & 378 & \\
\hline Laparoscopic proximal gastrectomy volume & 7.40 & 5.80 & $0^{*}$ & 84 & \\
\hline Number of average harvested lymph nodes & 27.40 & 27.18 & 8 & 62 & \\
\hline Liver metastasis proportion & $2.01 \%$ & $2.06 \%$ & $0 \%$ & $21.69 \%$ & Number of liver metastasis/gastrectomy volume \\
\hline Palliative surgery proportion & $2.06 \%$ & $1.87 \%$ & $0 \%$ & $12.39 \%$ & Palliative surgery volume/gastrectomy volume \\
\hline
\end{tabular}

Mean' indicates the weighted average value of the training dataset (2014-2015).

"Due to the fact that a few hospitals only conduct open gastrectomy or laparoscopic gastrectomy (with a few conversions), the number of 0 was reported, respectively.

TABLE 2 | Surgical safety and recovery outcomes.

\begin{tabular}{lcr}
\hline Outcomes (min. to max.) & Mean & Mean' \\
\hline Mortality, \% & $0.32(0-3.6)$ & 0.35 \\
Reoperation rate, \% & $1.46(0-7.08)$ & 1.55 \\
Postoperative stay, days & $15.69(7.50-26)$ & 16.10 \\
\hline
\end{tabular}

Mean' indicates the weighted average value of the training dataset (2014-2015).

our data supports the application of laparoscopy in gastric cancer patients, as the laparoscopic resection volume was identified as a protective factor. The advantages of the laparoscopic approach in colorectal surgery has now reached consensus among doctors, while its application in gastric cancer surgery remains in the early stage cases. The protective effect of the higher laparoscopic volume may be partly explained with the higher number of early stage cases in those centers, since the surgical procedures are standardized and face fewer technical difficulties. Similarly, the safety analysis of the CLASS-01 (Chinese Laparoscopic Gastrointestinal Surgery Study-01) trial has supported the use of laparoscopy in locally advanced gastric cancer cases (18), with similar oncological survival outcomes compared to the open procedures (19).

Higher liver metastasis proportion and higher palliative surgery proportion were identified as risk and protective factors, respectively, in our study. This is in agreement with the NCCN (National Comprehensive Cancer Network) and Japanese guidelines, which are, in general, against surgical therapy in M1 patients $(20,21)$. The surgical complexity and surgical trauma to multiple organs may explain the higher mortality in this type of patients. Yet, the Japanese guidelines also emphasize the possibility of hepatectomy for patients with a small number of metastatic nodules (20). A recent systematic review suggests better survival rates in favor of hepatectomy in this group of patients (22). With the recent advances in chemotherapy, more hepatectomies are performed for those gastric cancer patients with resectable nodules after conversion therapy. Our data address the short-term risks of this complex procedure, emphasizing the importance of safety evaluation when designing the surgical plan.

In addition to the aforementioned disease associated factors, our analysis also revealed many institutional factors which might influence mortality rates. Low GDP province and low annual volume seem to increase the in-hospital mortality of gastric cancer surgery. Similar results were also reported in the other literature $(23,24)$. One of the solutions for this is to relocate patients to select surgical theaters for complicated surgeries. Many European countries with low incidence of gastric cancer have applied this concept in practice by referring those patients to certain centers for gastrectomy (25). Greater volume often implies a greater level of technical competence 
TABLE 3 | Risk model of in-hospital mortality.

\begin{tabular}{|c|c|c|c|c|}
\hline Variables & Status & Parameter estimation & $95 \% \mathrm{Cl}$ & $P$-value \\
\hline Provincial GDP level & middle-low GDP & 0.97 & 0.37 to 1.57 & 0.002 \\
\hline Annual gastrectomy volume & $100-500$ & 0.75 & 0.10 to 1.40 & 0.002 \\
\hline Liver metastasis proportion & higher proportion & 0.42 & 0.25 to 0.59 & $<0.001$ \\
\hline Male patient proportion & higher proportion & 0.23 & 0.08 to 0.37 & 0.004 \\
\hline Reoperation rate & higher rate & 0.18 & 0.01 to 0.35 & 0.0380 \\
\hline Urgent surgery volume & Yes & 0.17 & 0.02 to 0.33 & 0.027 \\
\hline $\begin{array}{l}\text { Distal gastrectomy with B2 } \\
\text { reconstruction volume }\end{array}$ & higher volume & -0.32 & -0.54 to $(-0.10)$ & 0.005 \\
\hline Palliative surgery proportion & higher proportion & -0.32 & -0.53 to $(-0.12)$ & 0.003 \\
\hline Laparoscopic resection volume & higher volume & -0.36 & -0.69 to $(-0.03)$ & 0.037 \\
\hline
\end{tabular}

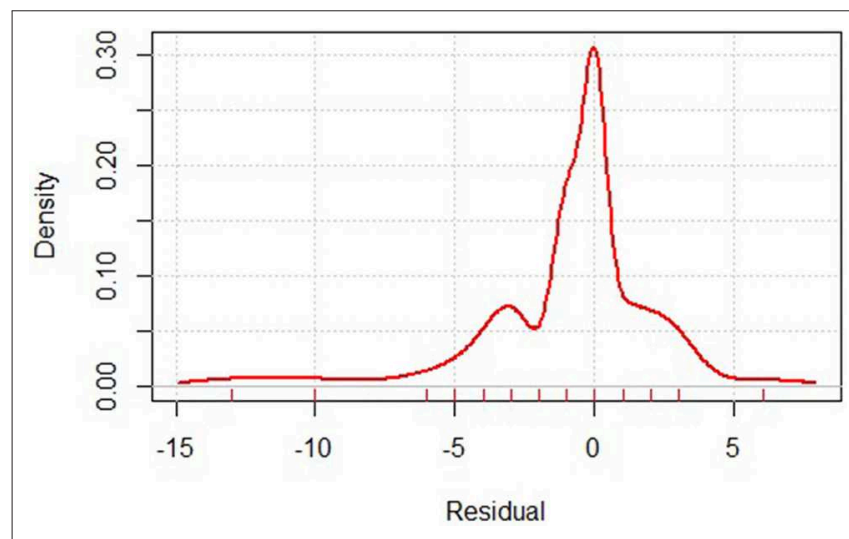

FIGURE 1 | Residual distribution of gastric cancer mortality prediction model. Residual calculates the difference between the actual and predicted value.

from surgeons, which may result in enhanced safety outcomes $(8,24,26)$. In addition, higher volume centers often indicate high-intensity intensive care units, the additional availability of multi-disciplinary teams and interventional radiology, effective prevention, and management of complications. These factors also influence the postoperative mortality (26). However, whether concentrating gastric cancer surgery fits the needs of the medical environment in China requires more research and discussion, given its large population and vast territories.

Distal gastrectomy was found to be a protective factor for mortality. This is in accordance with the literature reporting that distal gastrectomy is associated with fewer complications and thus lower in-hospital mortality when compared to the Japanese NCD data of distal and total gastrectomy $(3,4)$. Unfortunately, post-operative safety outcomes, i.e., complications, were not documented in a standardized manner in CGSD. It should be emphasized that morbidity remains substantial after gastrectomy. Our database also required complication rate data from each participant, but it varied between 0.3 and $33.1 \%$. This is mainly because of the lack of standardized complication registration in China. Given that our union was devoted to this subject and has published the first consensus for standardized complication diagnosis and registration in China (27), several on-going prospective cohort studies are expected to reveal the complication rate and its severity after gastric cancer surgery in China. We believe these efforts would result in better data quality and thus better surgical outcomes in the future.

Considering the overall number of centers performing gastrectomy in China, our database has collected a portion of them and the majority are tertiary (3A-level) hospitals, which inevitably introduced selection bias in the current research. However, given the fact that most gastric cancer cases are treated in 3A-level hospitals in China (17), our findings may prove valuable to health care policy makers in China. Our prediction model reached a satisfactory result in predicting mortality, in which most residuals in the model are concentrated near 0 , indicating nearly no difference between prediction and observation. This demonstrates its effectiveness. To reach a better sampling of the disease population, we are encouraging more hospitals to submit their data to the database. The number of participating centers continue to increase since its activation, and in 2018, CGSD has 85 participating members.

Another limitation to our study is that this institutional level database may not provide exclusive and conclusive answers to many detailed questions. Our analysis did not include any preoperative comorbidities or risk factors (e.g., weight loss or American Society of Anesthesiologists score), which could also influence surgical safety. The next step in data registration is to conduct individual level data collection which includes preoperative factors. The inclusion of such factors has begun in several centers in 2017, and the analysis of said individual data is on-going. It has been acknowledged that it is difficult to collect all details for a national database. Neither ACSNSQIP nor the Japanese NCD included disease-specific data (e.g., staging and pathological outcomes) in their databases (8). This is mainly subject to the purpose of their databases: surgical safety. Moreover, maintaining such large-scale databases is challenging (10). Improving our Chinese gastrointestinal union database by learning from our international peers is necessary to ensure its quality and cost-effectiveness. As the first application, our analysis of the gross data still delivers many important insights into the clinical practice.

In conclusion, we have reported the first risk stratification study for gastric cancer surgeries, using a nationwide institutional 
level database. The surgical outcomes were satisfactory. These identified risk factors could be used in reference for future therapy selection, while serving as additional insight for Chinese health care policy makers.

\section{DATA AVAILABILITY}

The datasets analyzed in this manuscript are not publicly available. Requests to access the datasets should be directed to ziyu_li@hsc.pku.edu.cn.

\section{AUTHOR CONTRIBUTIONS}

ZL, JJ, FS, and RM designed the study. The acquisition of data was conducted by ZW, HCh, FS, RM, JD, YSu, AX, YZ, YW, LC, YX, HCa, YH, ZX, MZ, MY, CH, JS, HLi, LF, JH, XH, GuolL, PY, GuoxL, YSh, HLu, YL, MX, TL, and ZL. The data analysis and interpretation was performed by $\mathrm{HCh}, \mathrm{ZW}, \mathrm{XY}$, and ZZ. ZW, $\mathrm{HCh}$, and TS drafted the manuscript. All authors extensively and critically revised the manuscript before submission.

\section{FUNDING}

This study was funded by the Beijing Municipal Science \& Technology project, Beijing Municipal Science \& Technology Commission (D131100005313010), and National Key Technology Research and Development Program of the Ministry of Science and Technology of China (2014BAI09B02).

\section{ACKNOWLEDGMENTS}

On behalf of the China Gastrointestinal Cancer Surgery Union, we thank all participating centers and data managers for their continued contribution toward this project. The participating centers include Affiliated Hospital of Guangdong Medical University, Affiliated Hospital of Guiyang Medical College, Affiliated Hospital of Xinjiang Medical University, Affiliated Hospital of Zunyi Medical University, Beijing Hospital, Cancer Hospital Chinese Academy of Medical Sciences, First Affiliated Hospital of Dalian Medical University, First Affiliated Hospital of Hainan Medical College, Hainan Medical University, First Affiliated Hospital of Kunming Medical University, Fourth Hospital of Hebei Medical University, Fudan University Shanghai Cancer Center, Fudan University

\section{REFERENCES}

1. Chen W, Zheng R, Baade PD, Zhang S, Zeng H, Bray F, et al. Cancer statistics in China, 2015. CA Cancer J Clin. (2016) 66:115-132. doi: 10.3322/caac.21338

2. Bartlett EK, Roses RE, Kelz RR, Drebin JA, Fraker DL, Karakousis GC. Morbidity and mortality after total gastrectomy for gastric malignancy using the American College of Surgeons National Surgical Quality Improvement Program database. Surgery. (2014) 156:298-304. doi: 10.1016/j.surg.2014.03.022

3. Kurita N, Miyata H, Gotoh M, Shimada M, Imura S, Kimura W, et al. Risk model for distal gastrectomy when treating gastric cancer
Zhongshan Hospital, Fujian Medical University Union Hospital, Fujian Provincial Cancer Hospital, Gansu People's Hospital, Gansu Provincial Cancer Hospital, General Hospital of Ningxia Medical University, Guangdong General Hospital, Guangdong Hospital of Traditional Chinese Medicine, Guangxi Medical University Affiliated Tumor Hospital, Harbin Medical University Cancer Hospital, Heji Hospital, Henan Cancer Hospital, Hunan Cancer Hospital, Jiangsu People's Hospital, Meizhou People's Hospital, Nanfang Hospital, Nanjing General Hospital, Ningbo First Hospital, Peking University Cancer Hospital, Peking University First Hospital, Peking University People’s Hospital, Penglai People's Hospital, People's Liberation Army General Hospital, PLA Army General Hospital, Qilu Hospital of Shandong University, Qingdao Municipal Hospital, Shandong Provincial Hospital, Shandong University Qilu Hospital, Shanghai Jiao Tong University Renji Hospital, Shanghai Jiao Tong University Ruijin Hospital, Sichuan Provincial People's Hospital, Sir Run Run Shaw Hospital, Southwest Hospital, Sun Yat-sen University Cancer Center, The 307th Hospital of the Chinese People's Liberation Army, The Affiliated Hospital of Inner Mongolia Medical University, The Affiliated Hospital of Qingdao University, The Affiliated Hospital of Qinghai University, The First Affiliated Hospital of Anhui Medical University, The First Affiliated Hospital of Xiamen University, The First Affiliated Hospital of Xi'an Jiaotong Hospital, The First Affiliated Hospital of Xingjiang Medical University, The First Hospital of China Medical University, The First Hospital of Jilin University, The First Hospital of Nanchang University, The Second Affiliated Hospital of Xi' an Jiaotong Hospital, The Second Clinical Hospital of Jilin University, The Sixth Affiliated Hospital of Sun Yat-sen University, The Third Clinical Hospital of Jilin University, Tianjin Medical University Cancer Institute and Hospital, Tianjin Medical University General Hospital, Weifang People's Hospital, West China Hospital, Wuhan Union Hospital, Xiangya Hospital, Xijing Hospital, Xingtai People's Hospital, Yunnan First People's Hospital, Zhejiang Cancer Hospital, Zhejiang Provincial Hospital of Traditional Chinese Medicine, and Zhongshan Hospital Xiamen University.

\section{SUPPLEMENTARY MATERIAL}

The Supplementary Material for this article can be found online at: https://www.frontiersin.org/articles/10.3389/fonc. 2019.00846/full\#supplementary-material 
6. Markar S, Gronnier C, Duhamel A, Mabrut JY, Bail JP, Carrere N, et al. The impact of severe anastomotic leak on long-term survival and cancer recurrence after surgical resection for esophageal malignancy. Ann Surg. (2015) 262:972-80. doi: 10.1097/SLA.0000000000001011

7. Hayashi T, Yoshikawa T, Aoyama T, Hasegawa S, Yamada T, Tsuchida K, et al. Impact of infectious complications on gastric cancer recurrence. Gastric Cancer. (2015) 18:368-74. doi: 10.1007/s10120-014-0361-3

8. Messager M, Pasquer A, Duhamel A, Caranhac G, Piessen G, Mariette C, et al. Laparoscopic gastric mobilization reduces postoperative mortality after esophageal cancer surgery: a French Nationwide Study. Ann Surg. (2015) 262:817-23. doi: 10.1097/SLA.0000000000001470

9. Suzuki H, Gotoh M, Sugihara K, Kitagawa Y, Kimura W, Kondo S, et al. Nationwide survey and establishment of a clinical database for gastrointestinal surgery in Japan: targeting integration of a cancer registration system and improving the outcome of cancer treatment. Cancer Sci. (2011) 102:22630. doi: 10.1111/j.1349-7006.2010.01749.x

10. Miyata $\mathrm{H}$, Gotoh $\mathrm{M}$, Hashimoto $\mathrm{H}$, Motomura $\mathrm{N}$, Murakami A, Tomotaki A, et al. Challenges and prospects of a clinical database linked to the board certification system. Surg Today. (2014) 44:1991-9. doi: 10.1007/s00595-013-0802-3

11. Ludwig DS, Currie J. The association between pregnancy weight gain and birthweight: a within-family comparison. Lancet. (2010) 376:98490. doi: 10.1016/S0140-6736(10)60751-9

12. Arellano M, Bond S. Some tests of specification for panel data: Monte Carlo evidence and an application to employment equations. Rev Econ Stud. (1991) 58:277-97. doi: 10.2307/2297968

13. Leggett L, Ball D. Future climate trends from a first-difference atmospheric carbon dioxide regression model involving emissions scenarios for business as usual and for peak fossil fuel. arXiv:1404.7469 (2014). Available online at: https://arxiv.org/abs/1404.7469

14. Lee H-J, Kim H-H, Han SU, Kim MC, Hyung WJ, Ryu SW, et al. Morbidity and mortality after laparoscopy-assisted and open distal gastrectomy for stage I gastric cancer: Results from a multicenter randomized controlled trial (KLASS-01). Am Soc Clin Oncol. (2015) 33:4. doi: 10.1200/jco.2015.33.3_suppl.4

15. Songun I, Putter H, Kranenbarg EM, Sasako M, van de Velde CJ. Surgical treatment of gastric cancer: 15-year follow-up results of the randomised nationwide Dutch D1D2 trial. Lancet Oncol. (2010) 11:43949. doi: 10.1016/S1470-2045(10)70070-X

16. Kobayashi H, Miyata H, Gotoh M, Baba H, Kimura W, Kitagawa Y, et al. Risk model for right hemicolectomy based on 19,070 Japanese patients in the National Clinical Database. J Gastroenterol. (2014) 49:104755. doi: 10.1007/s00535-013-0860-8

17. Sun XJ, Shi JF, Guo LW, Huang HY, Yao NL, Gong JY, et al. Medical expenses of urban Chinese patients with stomach cancer during 20022011: a hospital-based multicenter retrospective study. BMC Cancer. (2018) 18:435. doi: 10.1186/s12885-018-4357-y

18. Hu Y, Huang C, Sun Y, Su X, Xue Y, Hu J, et al. Laparoscopic D2 subtotal gastrectomy versus conventional open surgery for advanced gastric cancer: The safety analysis from a multicenter prospective randomized controlled trial in China (CLASS-01 trial). Am Soc Clin Oncol. (2015) 33:122. doi: 10.1200/jco.2015.33.3_suppl.122

19. Jiang Y, Huang C, Sun Y, Su X, Cao H, Hu J, et al. Effect of Laparoscopic vs Open Distal Gastrectomy on 3-Year Disease-Free Survival in Patients With Locally Advanced Gastric Cancer: The CLASS-01 Randomized Clinical Trial. Jama. (2019) 321:1983-92. doi: 10.1001/jama.2019.5359

20. Japanese Gastric Cancer Association. Japanese gastric cancer treatment guidelines 2014 (ver. 4). Gastric Cancer. (2017) 20:1-19. doi: 10.1007/s10120-016-0622-4

21. Ajani JA, D'Amico TA, Almhanna K, Bentrem DJ, Chao J, Das P, et al. Gastric cancer, version 3.2016, NCCN clinical practice guidelines in oncology. J Natl Compr Cancer Netw. (2016) 14:1286-312. doi: 10.6004/jnccn. 2016.0137

22. Liao YY, Peng NF, Long D, Yu PC, Zhang S, Zhong JH, et al. Hepatectomy for liver metastases from gastric cancer: a systematic review. BMC Surg. (2017) 17:14. doi: 10.1186/s12893-017-0215-0

23. Markar S, Gronnier C, Duhamel A, Bigourdan JM, Badic B, du Rieu MC et al. Pattern of postoperative mortality after esophageal cancer resection according to center volume: results from a large European multicenter study. Ann Surg Oncol. (2015) 22:2615-23. doi: 10.1245/s10434-0144310-5

24. Reames BN, Ghaferi AA, Birkmeyer JD, Dimick JB. Hospital volume and operative mortality in the modern era. Ann Surg. (2014) 260:24451. doi: 10.1097/SLA.0000000000000375

25. Nelen SD, Heuthorst L, Verhoeven RHA, Polat F, Kruyt PM, Reijnders $\mathrm{K}$, et al. Impact of centralizing gastric cancer surgery on treatment, morbidity, and mortality. J Gastrointest Surg. (2017) 21:2000-8. doi: 10.1007/s11605-017-3531-x

26. Tol JA, van Gulik TM, Busch OR, Gouma DJ. Centralization of highly complex low-volume procedures in upper gastrointestinal surgery. A summary of systematic reviews and meta-analyses. Dig Surg. (2012) 29:37483. doi: $10.1159 / 000343929$

27. Ji J. Chinese consensus on standardization of complication diagnosis and registration after gastrointestinal cancer surgery (ver. 2018). Chin J Pract Surg. (2018) 38:589-95. doi: 10.19538/j.cjps.issn1005-2208.2018.06.01

Conflict of Interest Statement: The authors declare that the research was conducted in the absence of any commercial or financial relationships that could be construed as a potential conflict of interest.

Copyright (C) 2019 Wu, Cheng, Shan, Ying, Miao, Dong, Sun, Xu, Zhou, Wang, Chen, Xue, Cao, Hua, Xu, Zheng, Yan, Huang, Suo, Liang, Fan, Hu, Hu, Li, Yu, Li, Shi, Luo, $\mathrm{Li}$, Xie, Liu, Zhang, Shi, Li and Ji. This is an open-access article distributed under the terms of the Creative Commons Attribution License (CC BY). The use, distribution or reproduction in other forums is permitted, provided the original author(s) and the copyright owner(s) are credited and that the original publication in this journal is cited, in accordance with accepted academic practice. No use, distribution or reproduction is permitted which does not comply with these terms. 